\title{
Novos medicamentos registrados no Brasil: podem ser considerados como avanço terapêutico?
}

\author{
New medicines registered in Brazil: \\ can they be considered as a therapeutic advance?
}

Cíntia M aria Gava ${ }^{1}$

Jorge Antonio Zepeda Bermudez ${ }^{2}$

Vera Lúcia Edais Pepe ${ }^{2}$

André Luiz Almeida dos Reis ${ }^{1}$

\footnotetext{
${ }^{1}$ Agência Nacional de

Vigilância Sanitária,

M inistério da Saúde. SIA,

Trecho 5, Área Especial 57.

71205-050 Brasília DF. cintia.gava@anvisa.gov.br

${ }^{2}$ Escola Nacional deSaúde

Pública Sergio Arouca,

Fundação Oswaldo Cruz.
}

Abstract Technological advances in the last century led to the discovery of a variety of medicines. Their introduction, not always with significant therapeutic gains and not without risk, made the register by regulatory agency an important moment in their evaluation. The aim of this study was to analyze 49 new medicines regi stered by the National Health Surveillance Agency (Anvisa), between the years 2000 and 2002 and commercialized in the first half of 2003, considering their therapeutic advantage. M ost medicines (42.9\%) were from the American pharmaceutical industry. M ost of them (63.3\%) were classified as a new molecular entity, followed by the new fixed dose combinations (28.6\%). Seventy-five percent were registered at the Food and Drug Administration (FDA). It was possible to obtain the classification of its N ew Drug Application (NDA) according to their therapeutic potential to 33 medicines and $57.6 \%$ of them wereclassified as standard. M ost of the new registered medicines did not provide any therapeutic advantage. It is suggested that Anvisa disseminate the analysis information on innovation, efficacy, safety, brought ( or not) by new drugs. This kind of information is important to consumers, health professionals and health managers. Key words $\mathrm{H}$ ealth surveillance, $\mathrm{N}$ ew drugs, M edicines, Register, Innovation, Pharmaceutical industry
Resumo Avanços tecnológicos no último século permitiram a descoberta de vários fármacos. Sua introdução, nem sempre com ganhos terapêuticos expressivos e não destituídos de riscos, faz do registro importante momento na avaliação de novos medicamentos. Foi analisado o registro de 49 novos medicamentos pela Agência $\mathrm{N}$ acional de Vigilância Sanitária entre 2000 e 2002 e comercializados no primeiro semestre de 2003, considerando 0 ganho terapêutico ante os tratamentos existentes. Predominou a produção por empresas americanas (42,9\%). A maioria dos medicamentos (63,3\%) foi classificada como entidade molecular nova, seguindo-se as novas associações (28,6\% ). Possuíam registro na Food and Drug Administration 75,5\% dos medicamentos, sendo possível obter a classificação de sua N ew D rug A pplication segundo o potencial terapêutico para 33 medicamentos, dos quais $57,6 \%$ foram classificados como padrão. Conclui-se que predomina o registro de medicamentos sem real vantagem terapêutica sobre os já existentes, fato também verdadeiro internacionalmente. São feitas sugestõesà Anvisa deforma a estabelecer e divulgar informações quanto à inovação, eficácia e segurança dos novos medicamentos, já que são importantes para o consumidor, os profissionais de saúde e os gestores.

Palavras-chave Vigilância sanitária, M edicamentos novos, M edicamentos, Registro, Inovação, Indústria farmacêutica 
Introdução

Os avanços tecnológicos no último século permitiram a descoberta e a introdução, no mercado, de uma grande variedade de medicamentos. Entretanto, passados os anos de grande inovação na indústria farmacêutica (1940-1960), houveum período de letargia no que se refere à descoberta de fármacos que representassem avanços terapêuticos efetivos, dando-se início a uma fase de lançamento de "novidades" que não passam de maquiagem feita em produtos já existentes no mercado ${ }^{1}$.

A estratégia da indústria farmacêutica é a manutenção de um mercado lucrativo, produzindo medicamentos muito similares aos campeões de venda. No mercado americano e também no brasileiro estão à venda, por exemplo, seis tipos de estatinas (sinvastatina, lovastatina pravastatina, fluvastatina, atorvastatina e rosuvastatina), usadas para a redução do colesterol, que são variantes da primeira estatina descoberta, a sinvastatina. Estas "novidades", com peque nas variações químicas entre si, também são conhecidos como me-too ${ }^{2}$.

A pesar de, na maioria dos casos, não agregarem ganho terapêutico ao tratamento com 0 medicamento lançado inicialmente, os medicamentos denominados me too são anunciados por seus fabricantes como medicamentos novos. 0 grande investimento em marketing para essas "novidades" acaba conquistando muitos profissionais de saúde e consumidores, que passam a prescrever eutilizar esses medicamentose, muito frequentemente, pressionam os sistemas de saúde para a sua incorporaçãa ${ }^{3}$.

Esse cenário constitui um dos principais desafios para os sistemas de saúde na atualidade e não está restrito ao mercado de medicamentos, estendendo-se para muitos outros tipos de produtos e serviços da área da saúde.

0 momento correspondente ao registro sanitário de medicamentos, sob responsabilidade das autoridades sanitárias em diversos países, pode ser considerado como uma importante oportunidade para a avaliação desses medicamentos novos. É nessemomento quese deveavaliar não apenas a eficácia ea segurança, mas também o ganho terapêutico representado por esses novos medicamentos.

Este estudo teve como objetivo analisar um conjunto de medicamentos novos registrados pela Agência N acional de Vigilância Sanitária (Anvisa) entre os anos de 2000 e 2002 e comercializados no primeiro semestre de 2003, enfocando 0 avanço terapêutico em relação aos medicamentos já existentes.

\section{M etodologia}

Trata-se de estudo realizado por meio de pesquisa documental, tendo como fonte de dados as páginas eletrônicas dos órgãos reguladores selecionados, Food and Drug Administration (FDA), dos Estados Unidos da América ${ }^{4}$, e Anvisa ${ }^{5}$, consultadas no período de outubro/2004 a março/2005.

A relação dos medicamentos novos registrados pela Agência N acional de Vigilância Sanitária entre os anos de 2000 e 2002 foi extraída do estudo realizado por Reis ${ }^{6}$ e é composta por medicamentos novos registrados pela Anvisa que estavam em comercialização no primeiro semestre de 2003. Os medicamentos foram analisados quanto: (1) ao país-sede da empresa fabricante; (2) à classificação segundo as definições de produto novo, presentes na Instrução Normativa no 1 da antiga Secretaria Nacional de Vigilância Sanitária (SNVS), de 30/9/19947, vigente atémarço de 2003; (3) ao registro na Food and Drug Administration (FDA); (4) à classificação segundo o potencial terapêutico definido pela FDA (prioritária ou padrão).

$\mathrm{Na}$ análise do tipo de registro concedido pela Anvisa, foi considerada a legislação vigente no período (2000-2002). Entretanto, vale ressaltar que, a partir de maio de 2003, passou a vigorar a Resolução da Diretoria Colegiada (RDC) $n=136^{8}$, que dispõe sobre o registro de medicamento novo. Esta última inclui, além dos casos já contemplados nal nstrução Normativa no 1, de 1994, os registros de "nova via de administração" e de "nova forma farmacêutica".

A FDA classifica os medicamentos segundo 0 avanço terapêutico durante a análise para concessão do registro. Esta foi a principal razão para a escolha do modelo de registro adotado naquela agência na análise e na caracterização do avanço terapêutico dos medicamentos registrados pela Anvisa. Nos Estados Unidos da América, as empresas farmacêuticas que desejam comercializar seus medicamentos devem solicitar ao órgão regulador, a FDA, uma autorização para aplicação de novo medicamento (NDA). Esta solicitação deve ser feita ao Centro de Avaliação e Pesquisa de M edicamentos (CDER) ou, quando se tratar de medicamentos biológicos, ao Centro de Avaliação e Pesquisa de Biológicos (CBER)9. Para o início do processo de avaliação de uma NDA, o CDER utiliza um sistema de classificação 
que permite diferenciar os níveis deinovação dos medicamentos. Esta classificação é feita a partir da caracterização do "tipo químico" e do "potencial terapêutico" dos medicamentos ${ }^{10}$. A classificação segundo o potencial terapêutico considera a"eficácia clínica" do medicamento quando comparada à eficácia demedicamentosjá disponíveis no mercado, destinados ao diagnóstico, tratamento ou prevenção da mesma doença, tendo por base as evidências disponíveis no período da avaliação. Se o medicamento não apresentar nenhuma vantagem clínica sobre os medicamentos já comercializados, o CDER denomina a avaliação desta NDA como padrão (S-standard). Ao contrário, se demonstrar avanço terapêutico, a avaliação da NDA pode ser classificada como "prioritária" (P-priority). 0 avanço terapêutico pode estar relacionado à maior eficácia; redução substancial de reações adversas que limitam o tratamento; aumento da adesão ao tratamento ou evidências de segurança e eficácia para uma nova subpopulação de pacientes ${ }^{10}$.

Na FDA, uma NDA classificada como prioritária deverá ter o seu processo de avaliação finalizado, em média, seis meses após a data de submissão pela empresa. Já uma NDA classificada como padrão deverá ser avaliada em, no mínimo, dez meses ou aproximadamente trezentos dias após a data de submissão na FDA ${ }^{11}$.

\section{Resultados}

O Quadro 1 apresenta a relação dos 49 medicamentos novos registrados pela Anvisa entre os anos de 2000 e2002 e comercial izados em 2003. A produção por empresas farmacêuticas com sede nos EUA mostrou-se predominante, representando $42,9 \%$ dos medicamentos analisados ( 21 medicamentos). Empresas com sede no Brasil e na Suíça foram responsáveis pela produção de $16,3 \%$ (oito) dos medicamentos, cada país, e $24,5 \%$ (12) medicamentos foram produzidos por empresas com sede em outros países europeus.

O Gráfico 1 apresenta a classificação dos medicamentos novos registrados pela Anvisa segundo as definições de produto novo, constantes na Instrução N ormativa SN VS nำ 1, de 1994. O bserva-se que $63,3 \%$ (31) dos medicamentos foram classificados como entidade molecular nova, seguindo-se as novas associações de $28,6 \%$ (14) dos medicamentos.

Quadro 1. M edicamentos novos registrados na Anvisa em 2000, 2001 e 2002, segundo classificações da Anvisa e da FDA.

\begin{tabular}{|c|c|c|c|c|}
\hline Medicamento & $\begin{array}{l}\text { País-sede do } \\
\text { fabricante }\end{array}$ & Classificação Anvisa & $\begin{array}{l}\text { Registro } \\
\text { FDA }\end{array}$ & $\begin{array}{l}\text { Classificação } \\
\text { FDA }\end{array}$ \\
\hline $\begin{array}{l}\text { ACETATO DE CASPOFUNGINA 50mg } \\
\text { pó liof inj }\end{array}$ & EUA & Entidade molecular nova & 2001 & NDA P \\
\hline $\begin{array}{l}\text { ACETATO DE CETRORELIX 0,25mg pó } \\
\text { liof inj }\end{array}$ & Suíça & Entidade molecular nova & 2000 & NDA S \\
\hline $\begin{array}{l}\text { ACETATO DE GANIRELIX } 0,5 \mathrm{mg} / \mathrm{ml} \text { sol } \\
\text { inj }\end{array}$ & Holanda & Entidade molecular nova & 1999 & NDA P \\
\hline $\begin{array}{l}\text { AMOXICILINA } 1.000 \text { mg + } \\
\text { SULBACTAM } 500 \text { mg - pó injetável }\end{array}$ & Alemanha & Nova associação & Não consta & Não consta \\
\hline BIMATOPROST $0,3 \mathrm{mg} / \mathrm{ml}$ sol oft & EUA & Entidade molecular nova & 2001 & NDA P \\
\hline $\begin{array}{l}\text { BUTIRATO DE HIDROCORTISONA } \\
1 \mathrm{mg} / \mathrm{g} \text { creme - } 15 \mathrm{~g}\end{array}$ & Brasil & $\begin{array}{l}\text { Sal novo, embora a } \\
\text { entidade molecular } \\
\text { correspondente já tenha } \\
\text { sido autorizada }\end{array}$ & 2002 & NDA S \\
\hline CILOSTAZOL 50mg - comp & Brasil & Entidade molecular nova & 2004 & NDA S \\
\hline $\begin{array}{l}\text { CLORIDRATO DE APOMORFINA } 2 \mathrm{mg} \\
\text { comp sublingual }\end{array}$ & EUA & Entidade molecular nova & Não consta & Não consta \\
\hline $\begin{array}{l}\text { CLORIDRATO DE PIOGLITAZONA } \\
\text { 15mg - comp }\end{array}$ & EUA & Entidade molecular nova & 1999 & NDA P \\
\hline
\end{tabular}


Quadro 1. continuação

\begin{tabular}{|c|c|c|c|c|}
\hline Medicamento & $\begin{array}{l}\text { País-sede do } \\
\text { fabricante }\end{array}$ & Classificação Anvisa & $\begin{array}{l}\text { Registro } \\
\text { FDA }\end{array}$ & $\begin{array}{l}\text { Classificação } \\
\text { FDA }\end{array}$ \\
\hline DESLORATADINA 5mg comp & EUA & Entidade molecular nova & 2002 & NDA S \\
\hline $\begin{array}{l}\text { ESOM EPRAZOL MAGNÉSIO } \\
\text { TRIIDRATADO 20mg - comp }\end{array}$ & Suécia & Entidade molecular nova & 2001 & NDA S \\
\hline $\begin{array}{l}\text { ESTRADIOL + NORGESTIM ATO } 1 \mathrm{mg} \\
\text { comp rosa / } 1 \mathrm{mg}+90 \mathrm{mcg} \text { comp branco }\end{array}$ & EUA & N ova associação & 1999 & NDA S \\
\hline $\begin{array}{l}\text { ETABONATO DE LOTEPREDNOL } 5 \mathrm{mg} / \mathrm{ml} \\
\text { sus oft - } 5 \mathrm{ml}\end{array}$ & EUA & Entidade molecular nova & 1998 & NDA S \\
\hline $\begin{array}{l}\text { ETONOGESTREL 68mg solução } \\
\text { dermatológica - implante }\end{array}$ & Holanda & Entidade molecular nova & Não consta & Não consta \\
\hline ETORICOXIB 60mg - comp & EUA & Entidade molecular nova & Não consta & Não consta \\
\hline EXEM ESTANO 25mg drágeas & Suécia & Entidade molecular nova & 1999 & NDA SV \\
\hline $\begin{array}{l}\text { FLUTRIM AZOL 0,01g/ml solução tópica } \\
\text { spray }\end{array}$ & Brasil & Entidade molecular nova & Não consta & Não consta \\
\hline $\begin{array}{l}\text { FUMARATO DE FORMOTEROL + } \\
\text { BUDESONIDA } 6 / 100 \text { mcg dose }\end{array}$ & Suécia & N ova associação & Não consta & Não consta \\
\hline INFLIXIMAB 10mg/ml po liof & EUA & Entidade molecular nova & 1998 & CBER \\
\hline INSULINA ASPART $100 \mathrm{UI} / \mathrm{ml}$ - 10ml & Dinamarca & $\begin{array}{l}\text { Alteração na concentração } \\
\text { de substância ativa ou de } \\
\text { propriedades }\end{array}$ & 2000 & NDA S \\
\hline $\begin{array}{l}\text { IRBESARTANA } 150 \mathrm{mg}+ \\
\text { HIDROCLOROTIAZIDA 300mg comp }\end{array}$ & França & N ova associação & 1997 & NDA S \\
\hline $\begin{array}{l}\text { ISOTRETINOÍNA } 0,5 \mathrm{mg} / \mathrm{g}+\text { ÁCIDO } \\
\text { SULFÔNICO BENILBENZIMIDAZÓLICO } 40 \\
\text { mg/g + BUTILMETOXID } \\
\text { IBENZOILMETANO } 15 \mathrm{mg} / \mathrm{g}+ \\
\text { OCTILMETOXICINAMATO } 80 \mathrm{mg} / \mathrm{g}- \\
\text { creme }\end{array}$ & EUA & N ova associação & Não consta & Não consta \\
\hline $\begin{array}{l}\text { LOPINAVIR + RITONAVIR 133,3mg + } \\
\text { 33,3mg cap gel mole }\end{array}$ & EUA & $\begin{array}{l}\text { Duas ou mais substâncias } \\
\text { ativas não registradas, } \\
\text { juntas em um mesmo } \\
\text { produto }\end{array}$ & 2000 & NDA P \\
\hline LUTROPINA ALFA (R-LH) 75UI pó liófilo inj & Suíça & Entidade molecular nova & 2004 & NDA S \\
\hline $\begin{array}{l}\text { MALEATO DE ENALAPRIL } 2,5 \mathrm{mg}+ \\
\text { BESILATO DE ANLODIPINO } 10 \mathrm{mg} \text { - cap } \\
\text { gel dura }\end{array}$ & Brasil & N ova associação & Não consta & Não consta \\
\hline $\begin{array}{l}\text { MALEATO DE METFORMINA } 250 \mathrm{mg}+ \\
\text { GLIBENCLAM IDA } 1,25 \mathrm{mg} \text { comp }\end{array}$ & Alemanha & N ova associação & 2000 & NDA S \\
\hline $\begin{array}{l}\text { MESILATO DE IMATINIB 50mg - cap gel } \\
\text { dura }\end{array}$ & Suíça & Entidade molecular nova & 2003 & NDA PV \\
\hline $\begin{array}{l}\text { MONONITRATO DE ISOSSORBIDA 60mg } \\
\text { + ACIDO ACETILSALICÍLICO 75mg - cap } \\
\text { gel }\end{array}$ & Brasil & N ova associação & Não consta & Não consta \\
\hline NATEGLINIDA 60mg - comp & Suíça & Entidade molecular nova & 2000 & NDA S \\
\hline
\end{tabular}




\begin{tabular}{|c|c|c|c|c|}
\hline \multicolumn{5}{|l|}{ Quadro 1. continuação } \\
\hline Medicamento & $\begin{array}{l}\text { País-sede do } \\
\text { fabricante }\end{array}$ & Classificação Anvisa & $\begin{array}{l}\text { Registro } \\
\text { FDA }\end{array}$ & $\begin{array}{l}\text { Classificação } \\
\text { FDA }\end{array}$ \\
\hline $\begin{array}{l}\text { OM EPRAZOL 20mg cap +AZITROMICINA } \\
\text { 500mg comp rev + AM OXICILINA 500mg } \\
\text { cap }\end{array}$ & Brasil & Nova associação & Não consta & Não consta \\
\hline OSELTAM IVIR 75mg - cap gel dura & Suíça & Entidade molecular nova & 1999 & NDA P \\
\hline $\begin{array}{l}\text { PEGINTERFERON ALFA } 2 \text { B } 50 \mathrm{mcg} / 0,5 \mathrm{ml}- \\
\text { pó liof inj }\end{array}$ & EUA & Entidade molecular nova & 2001 & CBER \\
\hline PIMECROLIMUS 10mg/g creme - 15g & Suíça & Entidade molecular nova & 2001 & NDA S \\
\hline $\begin{array}{l}\text { POLIVITAM INAS (Vitaminas B12/B6/B1) } \\
0,25 \mathrm{mg}+50 \mathrm{mg}+50 \mathrm{mg}+\text { DICLOFENACO } \\
\text { SÓDICO 50mg comp }\end{array}$ & Alemanha & Nova associação & Não consta & Não consta \\
\hline $\begin{array}{l}\text { QUINUPRISTINA + DALFOPRISTINA } \\
\text { 150mg + 350mg pó liof inj }\end{array}$ & França & $\begin{array}{l}\text { Duas ou mais substâncias } \\
\text { ativas não registradas, } \\
\text { juntas em um mesmo } \\
\text { produto }\end{array}$ & 1999 & NDA P \\
\hline RISEDRONATO SÓDICO 5mg - comp & EUA & Entidade molecular nova & 1998 & NDA S \\
\hline SIROLIM US 1mg/ml solução oral & EUA & Entidade molecular nova & 1999 & NDA S \\
\hline $\begin{array}{l}\text { SULFATO DE ABACAVIR } 300 \mathrm{mg}+ \\
\text { LAM IVUDINA 150mg + ZIDOVUDINA } 300 \\
\text { mg comp }\end{array}$ & EUA & Nova associação & 2000 & NDA P \\
\hline $\begin{array}{l}\text { SULFATO DE GENTAMICINA } 50 \mathrm{mg} / \mathrm{ml}+ \\
\text { DEXAMETASONA } 1,0 \mathrm{mg} / \mathrm{ml} \text { sol ocular } 5 \mathrm{ml}\end{array}$ & EUA & Nova associação & Não consta & Não consta \\
\hline $\begin{array}{l}\text { SULFATO DE GLICOSAMINA } 200 \mathrm{mg} / \mathrm{ml}+ \\
\text { CLORIDRATO DE LIDOCAIINA } 5 \mathrm{mg} / \mathrm{ml} \text { - } \\
\text { sol inj }\end{array}$ & Brasil & Nova associação & Suplemento & $\begin{array}{l}\text { Suplemento } \\
\text { nutricional }\end{array}$ \\
\hline $\begin{array}{l}\text { SULFATO DE GLICOSAMINA 250mg - cap } \\
\text { gel dura }\end{array}$ & Brasil & Entidade molecular nova & Suplemento & $\begin{array}{l}\text { Suplemento } \\
\text { nutricional }\end{array}$ \\
\hline TEGASERODE 2mg - comp & Suíça & Entidade molecular nova & 2002 & NDA P \\
\hline TELITROMICINA 400mg - comp & EUA & Entidade molecular nova & 2004 & NDA S \\
\hline $\begin{array}{l}\text { TELMISARTAN } 40 \mathrm{mg}+ \\
\text { HIDROCLOROTIAZIDA } 12,5 \mathrm{mg} \text { comp }\end{array}$ & Alemanha & Nova associação & 2000 & NDA S \\
\hline TEM OZOLOM IDA 5mg cap gel dura & EUA & Entidade molecular nova & 1999 & NDA PV \\
\hline TRAVOPOST 0,004\% sol oftálmica & EUA & Entidade molecular nova & 2001 & NDA P \\
\hline VERTEPORFIRINA 15mg pó liof - $826 \mathrm{mg}$ & Suíça & Entidade molecular nova & 2000 & NDA P \\
\hline $\begin{array}{l}\text { ZALEPLOM MICRONIZADO } 5 \mathrm{mg} \text { cap gel } \\
\text { dura }\end{array}$ & EUA & Entidade molecular nova & 1999 & NDA S \\
\hline ZANAMIVIR 5mg cap inalação & EUA & Entidade molecular nova & 1999 & NDA P \\
\hline
\end{tabular}

NDA S - Aplicação denovo medicamento classificada como padrão (S-standard) no FDA.

NDA P - A plicação de novo medicamento classificada como prioritária (P-priority) no FDA.

NDA PV - A plicação de novo medicamento classificada como prioritária para medicamento órfão no FDA.

NDA SV - Aplicação denovo medicamento classificada como padrão para medicamento órfão no FDA.

CBER - M edicamentos biológicos submetidos ao Centro de Avaliação e Pesquisa de Biológicos (CBER). 
Quanto ao registro na FDA, verificou-se que, dos 49 medicamentos registrados na Anvisa, $75,5 \%$ (37) estavam registrados na FDA. Foi possível a obtenção da classificação segundo o potencial terapêutico definido pela FDA para 33 medicamentos: $57,6 \%$ (19) tiveram a avaliação de sua NDA classificada como padrão (S) e $42,4 \%$ (14) tiveram sua NDA classificada como avaliação prioritária (P) (Gráfico 2).

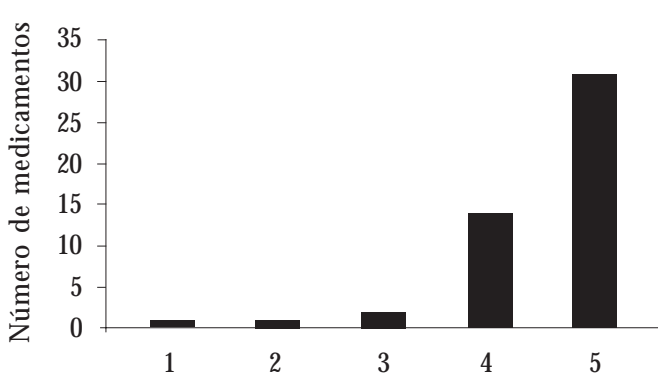

Classificação de produto novo

1 - Alteração na concentração de substância ativa ou de propriedades farmacocinéticas

2 - Sal novo, embora a entidade molecular correspondente já tenha sido autorizada

3 - Duas ou mais substâncias ativas registradas, juntas em um mesmo produto

4 - Nova associação

5 - Entidade molecular nova

Gráfico 1. Distribuição dos medicamentos segundo a classificação de produto novo da Instrução N ormativa SVS no 1. de 30/09/1994.

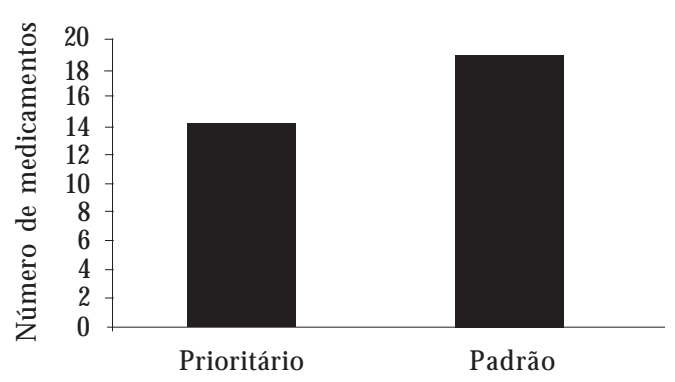

Classificação da NDA

Gráfico 2. Distribuição dos medicamentos segundo a classificação da NDA pelo potencial terapêutico na FDA.
A comparação dos gráficos 1 e 2 permiteconcluir que, apesar de a mai oria dos medicamentos novos serem classificados como nova entidade molecular pela Anvisa, a maior parte teve a avaliação de sua N DA classificada como padrão pela FDA. M esmo quando se consideram apenas os medicamentos que contêm novas entidades moleculares (24), 13 foram classificados com NDA padrão (S) e 11 foram classificados com NDA prioritária (P). Desta forma, apesar de a maioria dos medicamentos serem compostos por novas moléculas, o avanço terapêutico não foi significativo se comparado ao potencial terapêutico dos medicamentos já comercializados.

$\mathrm{N}$ ão foi constatado registro na FDA para quatro dos oito medicamentos fabricados por empresas brasileiras, sendo dois deles classificados com NDA padrão e dois como suplementos nutricionais. 0 mesmo foi verificado para as empresas americanas, pois os resultados obtidos indicam que dos vinte medicamentos produzidos por empresas americanas, menos da metade ( oito medicamentos) foi classificada com NDA prioritária.

Outro resultado a ser considerado éo fato de que 16 (33\%) dos 49 medicamentos analisados são associações de duas ou mais substâncias ( $Q$ uadro 1), sendo que dois deles contêm quatro substâncias ativas. Além disso, para esses dois medicamentos, produzidos por empresas americana ealemã, não foram identificados registros naFDA.

\section{Discussão}

Este estudo analisou 49 medicamentos novos re gistrados no Brasil, no período de 2000 a 2002, e comercializados no ano de 2003. Estudo publicado por Vidotti et al. ${ }^{12}$ apresenta um número maior de medicamentos novos registrados no Brasil, no mesmo período, totalizando 85 medicamentos. Entretanto, ao contrário do presente estudo, nem todos os medicamentos relacionados no estudo de Vidotti et al. ${ }^{12}$ estavam em comercialização em 2003.

Confirmaram-se o destaque americano e europeu no setor farmacêutico e a importância dessas empresas multinacionais no mercado farmacêutico brasileiro. Os EUA possuem uma posição destacada na produção de medicamentos e uma forte atuação comercial. Estudo publicado por Baxendale et al. ${ }^{13}$ aponta que, em 2005, o volumemundial de ven das demedicamentos (incluindo medicamentos over-the-counter) foi de 
aproximadamenteUS\$287,8 bilhões, eque a indústria farmacêutica americana contribuiu com aproximadamente US\$135,5 bilhões, representando $47 \%$ do total.

$\mathrm{Na}$ análise da racionalidade terapêutica das associações medicamentosas registradas no período, dois medicamentos merecem destaque, visto conterem quatro fármacos associados. É importante destacar que esses medicamentos foram registrados em 2000 e 2001, antes da publicação da Resolução RDC nํ 136, de 29 demaio de 2003, norma atualmente vigente que também trata do registro de associações medicamentosas ${ }^{8}$. Esta resolução permite a associação de, no máximo, três princípios ativos na mesma formulação por apresentação oral ou injetável, sendo aceita para as apresentações orais até quatro fármacos, se um deles for a cafeína. Ela também estabeleceque, para o registro de associações de fármacos, sejam apresentados estudos de biodisponibilidade relativa entre os princípios ativos associados e cada princípio ativo isolado, bem como ensaios clínicos controlados, provando que associações com as mesmas doses dos fármacos isolados tenham um efeito aditivo ou sinérgico, sem aumento dos riscos. Não há, na norma atual, referência a medicamentos de uso dermatológico, que é o caso de uma das associações apresentadas.

Ainda que a maior parte dos novos medicamentos registrados tenha sido classificada como entidade molecular nova ao serem avaliados quanto ao potencial terapêutico definido pela FDA, o maior número não apresentou avanço terapêutico em relação aos medicamentos já comercializados que justificasse prioridade na avaliação de suas NDA. Isto significa que, apesar de a maioria dos medicamentos serem compostos por novas moléculas, o ganho terapêutico trazido por esses medicamentos não ésignificativo se comparado ao potencial terapêutico dos medicamentos já comercializados.

Esses resultados podem ser reforçados pelo estudo publicado por Baxendaleet al. ${ }^{13}$ segundo os quais a descoberta de novos fármacos tem sido tarefa cada vez mais difícil e os gastos com pesquisa edesenvolvimento ( $P \& D$ ) cada vez maiores. N esse sentido, muitas empresas farmacêuticas estão direcionando suas atividades para a reformulação de medicamentos já em comercialização, euma dessas estratégias éo investimento nos medicamentos me-too.

No que se refere aos gastos com $P \& D$, existe uma divergência entre os números anunciados pela indústria farmacêutica e aqueles apresentados por algumas instituições não governamen- tais. Dados baseados nos anos de 2000 a 2002 indicam que o lançamento de um novo medicamento, naquele período, custava em torno de US\$ 900 milhões $^{14}$. Segundo análise realizada pela Pharmaceutical Research and $M$ anufacturers of America (PhRMA), entidade que representa as empresas farmacêuticas e de biotecnologia americanas, a maior parte do aumento nos custos com $P \& D$ pode ser atribuída à extensão dos prazos para desenvolvimento dos medicamentos ( 10 a 15 anos), grandemente influenciada pelas exigências regulatórias atuais, relativas ao baixo risco sanitário. Entretanto, instituições independentes da indústria farmacêutica estimam que esses valores encontram-sesuperestimados, uma vez que incluem o custo de oportunidade e não levam em conta a inflação e a dedução de impostos, o que reduziria os custos para o lançamento de um novo medicamento para cerca de US\$110 milhões ${ }^{15}$.

0 registro sanitário de medicamento é o momento no qual os órgãos reguladores procedem à avaliação dos aspectos relacionados à eficácia, segurança e qualidade da nova tecnologia. É por meio dessa atividade que as autoridades sanitárias devem atuar como mediadoras entre os interesses dos fabricantes de medicamentos e as necessidades da saúde pública, visando, sobretudo, ao dever de proteção da saúde.

Para Garattini ${ }^{16}$, durantea análise para aprovação do registro deum medicamento novo, além dos atributos relacionados à qualidade, à segurança eà eficácia, deveria ser considerado o ganho terapêutico, seja em termos demaior eficácia, seja de maior segurança, maior adesão ao tratamento ou menor preço.

Em documento disponível no sítio el etrônico da Anvisa" ${ }^{17}$, intitulado "Posicionamento da Anvisa quanto ao registro de medicamentos novos considerados como metoos", são apresentados alguns argumentos para o fato de a Agência não poder negar o registro para esse tipo de medicamento em respostas a algumas correntes que defendem que medicamentos desse tipo nada acrescentam ao que já é disponível comercialmente, contribuindo para um aumento demedicamentos no mercado e para a exposição da população a riscos desnecessários.

Nesse sentido, ainda que o indeferimento do registro para esse tipo de medicamento não encontre amparo na legislação brasileira vigente, a classificação de tais medicamentos segundo as características químicas e o ganho terapêutico parece ser atividade factível durante o processo de registro. 
Por meio da Resolução RDC no $28^{18}$, de 4 de abril de 2007, a Anvisa instituiu a priorização da análise técnica das solicitações de regi stro (e pósregistro) de medicamentos, quando caracterizada a relevância pública. Conforme prevêa Resolução, essa priorização poderá ser solicitada pe las empresas e é destinada: (1) aos medicamentos que fazem parte dos elencos estabelecidos e publicados pelo M inistério da Saúde para suprir seus programas no âmbito do Sistema Ú nico de Saúde (SUS), compreendendo os medicamentos excepcionais e vacinas; (2) aos medicamentos utilizados para profilaxia ou tratamento de doença negl igenciada (medicamento órfão), ou doença emergente ou reemergente. As solicitações de priorização de análise são submetidas a uma comissão técnica da Gerência-Geral de M edicamentos (GGM ED) para a sua validação, e aquelas que não se enquadram nos critérios estabele cidos para a priorização da análise são analisadas segundo a ordem cronológica de entrada na Anvisa. Essa priorização da análise dos registros poderia ser um primeiro passo para o início da análise do ganho terapêutico dos medicamentos durante o processo de registro na Anvisa. É importante destacar que a classificação segundo 0 potencial terapêutico da FDA também é dada antes do início do processo de avaliação do dossiê de registro e é usada para definir o prazo de avaliação dos medicamentos.

No Brasil, a atuação do órgão sanitário federal torna-se ainda mais importante, visto ser o Estado brasileiro constitucionalmenteresponsável pela prestação da assistência à saúde, incluindose aí a assistência farmacêutica. Cabe ao Sistema Ú nico de Saúde a regulação sanitária dos medicamentos, por meio do Sistema $\mathrm{Nacional}$ de Vigilância Sanitária, bem como a provisão de medicamentos, por meio da Secretaria de Ciência, Tecnologia e Insumos Estratégicos, do Ministé rio da Saúde, e das Secretarias de Saúde dos estadose municípios.

Dentre os 49 medicamentos analisados, constantes no Quadro 1, foi possível identificar alguns medicamentos incorporados em programas do M inistério da Saúde, devendo ser distribuídos gratuitamente à população brasileira. D entre esses programas encontram-se o Programa Nacional de D oenças SexualmenteTransmissíveis e Aids (PN DST/Aids) e o na época denominado, para fins de financiamento entre as esferas de governo, Componente de M edicamentos de Dispensação Excepcional.

Ainda que no presente trabalho tenham sido utilizados dados de registros de medicamentos concedidos pela Anvisa há oito anos, o resultado obtido em relação ao avanço terapêutico é tema bastante atual. A partir de 2004, a Anvisa, como Secretaria Executiva da Câmara de Regulação do Mercado de Medicamentos (CM ED), passou a considerar o valor terapêutico dos novos medicamentos para apuração dos preços desses produtos, atividade realizada após a concessão do registro. As avaliações realizadas até 2009 demonstraram que mais de $80 \%$ dos novos medicamentos foram classificados como categoria II, ou seja, não apresentavam vantagem terapêutica ante os medicamentos já existentes no país para a mesma indicação terapêutica ${ }^{19}$.

Este é um debate atual também na Europa, sobretudo com a retirada de fármacos como rimonabanto e rofecoxibe do mercado internacional. No que diz respeito ao registro de novos fármacos, pode-se citar a análise do valor terapêutico dos medicamentos registrados na França feita regularmente pela revisa Prescrire International ${ }^{20}$. A aplicação dessa escala aos 359 medicamentos registrados entre os anos de 1999 e 2004 levou ao seguinteresultado: nenhum medicamento foi considerado um grande avanço terapêutico ("bravo"); apenas 3\% foram considerados "um avanço real", $12 \%$ considerados como "oferece um avanço", 26\% "possivelmente útil", $46 \%$ "não constituiu avanço" e $7 \%$ foram considerados "não aceitável". Em 2009, análise de 104 novos registros/novas indicações de fármacos já registrados na França resultou em apenas três fármacos com vantagem terapêutica sobre os fármacos já existentes, enquanto 19 apresentavam relação benefício-risco desfavorável ${ }^{21}$.

Ainda nesse sentido, documento elaborado por grupos de familiares, pacientes e profissionais de saúde da Europa ${ }^{22}$ aborda a necessidade dea European M edicines Agency (EM A), em seu plano de ação até2015, garantir sua independência intel ectual efinanceira em relação à indústria farmacêutica e de que o avanço terapêutico ante os tratamentos já disponíveis, demonstrado por meio de evidências científicas, seja um dos crité rios na avaliação para autorização de comercialização de qualquer medicamento.

0 estabelecimento da eficácia e segurança, bem como do real avanço terapêutico dos novos medicamentos e sua ampla divulgação são fundamentais para informar os profissionais de saúde sobre os reais ben efícios de um tratamento. A solicitação e a concessão de medicamentos pela viajudicial, tendo praticamente como única base a prescrição de medicamentos, constituem uma prática atualmente crescente no Brasil. Tal fato 
reforça ainda mais a importância de os prescritores conhecerem os riscos aos quais estão expondo seus pacientes e os reais ben efícios trazidos pelos novos fármacos ${ }^{23}$.

Uma das formas utilizadas para divulgação dos novos medicamentos à classe médica, estimulando o uso de medicamentos em indicações não aprovadas no registro, tem sido o financiamento da educação continuada dos prescritores $^{24}$. Diante desse cenário de crescentes desafios e interesses diversos, a informação fidedigna e independente do setor produtivo é uma ferramenta imprescindível para a prescrição racional de medicamentos e para a proteção da saúde dos cidadãos.

\section{Consideraçõesfinais}

A pesquisa realizada pode concluir que o perfil de novos medicamentos registrados e comercializados no Brasil, no período de 2000 a 2002, é majoritariamente de entidades moleculares novas. Ainda que seja esta a classificação dos novos produtos na agência reguladora brasileira quando de seu registro, não épossível afirmar queeles se constituam em inovações terapêuticas consideradas como prioritárias e, portanto, que re presentem significativo avanço terapêutico ante os medicamentos já existentes.

Considerando as questões e os resultados apresentados neste estudo, bem como as recomendações que os grupos europeus independentes sugerem a seu órgão regulador, pode-se elencar algumas sugestões à Anvisa, visando aprimorar sua atual forma de avaliação e concessão de registro de novos medicamentos.

A primeira delas é estabelecer a classificação dos medicamentos segundo a natureza química e 0 avanço terapêutico durante a avaliação para concessão do registro e não somentena etapa de definição de preço do medicamento, como faz a FDA. Ainda que não possa, segundo as normas atuais, negar o registro de medicamentos que potencialmente não trarão nenhum ganho para tratamento das enfermidades para as quais se destinam, a análise quanto à inovação trazida (ou não) por esses medicamentos, no que se refere a aspectos como eficácia, segurança e adesão ao tratamento, precisa ser iniciada (e divulgada) na etapa de registro do medicamento.

A segunda delas é a melhoria da divulgação, por meio do sítio eletrônico, dos atos referentes ao registro sanitário de medicamentos. $\mathrm{Nisso}$ podeser incluída a divulgação dos prazos de aná- lise, dos pareceres de indeferimento ou deferimento dos registros, da classificação dada ao registro (se medicamento novo, similar, genérico, etc.) e das bulas aprovadas. A disponibilização dessas informações para consulta pública poderá contribuir para maior transparência e legitimidade no processo de registro.

A última sugestão refere-se à divulgação das informações sobre a inovação, a eficácia e a segurança dos novos medicamentos de forma contínua e sistemática. Elas são importantes para 0 público consumidor de medicamentos, para os profissionais desaúdee, fundamentalmente, para os gestores do sistema de saúde, que poderão utilizar esses dados para embasar decisões como aquelas referentes à definição de preços desses medicamentos eà incorporação dessas novas tecnologias no sistema de saúde brasileiro.

\section{Colaboradores}

CM Gava foi a responsável pelo desenho e execução da íntegra da pesquisa, bem como pela redação de todas as versões do artigo; JAZ Bermudez participou na orientação da pesquisa, na discussão dos resultados e na discussão e revisão final do artigo; VLE Pepe participou na orientação da pesquisa, na análise dos dados e na revisão de todas as versões do artigo.

\section{Agradecimentos}

Os autores agradecem ao Centro Colaborador deVigilância Sanitária da Escola N acional deSaúdePública Sergio Arouca eà Agência $\mathrm{N}$ acional de Vigilância Sanitária pelo financiamento de parte da pesquisa. 


\section{Referências}

1. Barros JAC. Propaganda de medicamentos: atentado à saúde? São Paulo: Hucitec, Sobravime; 1995.

2. Angell $M$. The truth about the drug companies: how they deceive us and what to do about it. New York: Random House; 2004.

3. Lexchin J, Bero LA, Djulbegovic B, Clark O. Pharmaceutical industry sponsorship and research outcome and quality: systematic review. British M edical Journal 2003; 326:1167-1170.

4. EUA. Food and Drug Administration. Center for Drug Evaluation and Research. [site da Internet] [acessado 2009 jun 16]. Disponível em: http:// www.fda.gov/D rugs/default.htm

5. Brasil. Agência Nacional de Vigilância Sanitária. [site da Internet] [acessado 2009 jun 16] Disponível em: http://www.anvisa.gov.br

6. Reis ALA. N ovos produtos no mercado farmacêutico: padrão de difusão e preços [tese]. Rio de Janeiro: Escola Nacional de Saúde Pública Sergio Arouca, Fundação Oswaldo Cruz; 2004.

7. Brasil. Instrução Normativa $n-1$, de 30 de setembro de 1994. Estabelece os documentos necessários para processos de petições junto à Secretaria de Vigilância Sanitária. Diário Oficial da União 1994; 4 out.

8. Brasil. Resolução RDC № 136, de 29 de maio de 2003. Aprova o Regulamento Técnico para M edicamentos Novos com Princípios Ativos Sintéticos ou Semi-Sintéticos. Diário Oficial da União 2007; 5 abr.

9. EUA. Food and Drug Administration. The CDER Handbook. [acessado 2009 jun 16] Disponível em: http://www.fda.gov/cder/handbook/ndabox.htm

10. National Institute for $\mathrm{H}$ ealth $\mathrm{C}$ are $\mathrm{M}$ anagement. Changing Patterns of Pharmaceutical Innovation, 2002. [site da Internet] [acessado 2009 jun 16] Disponível em: http://www.nihcm.org/innovations.pdf

11. Meadows M. The FDA's drug review process: ensuring drugs are safe and effective. FDA Consumer Magazine 2002. [site da Internet] [acessado 2009 jun 16] Disponível em: http://www.fda.gov/fdac/ features/2002/402_drug.html

12. Vidotti CCF, Castro LLC, Calil SS. New drugs in Brazil: do they meet Brazilian public health needs? Rev Panam Salud Publica 2008; 24(1):36-45.

13. Baxendale IR, H ayward JJ, Ley SV, Tranmer GK. Pharmaceutical strategy and innovation: an academic perspective. Chem M ed Chem 2007; (2):768-788.

14. Gilbert J, Henske P, Singh A. Rebuilding Big Pharma's Business Model. In Vivo The Business and M edicine Report 2003; 21:73-80.

15. Public Citizen. $R x R \& D$ myths: the case against the drug industry's R\&D "scare card". Congress Watch July 2001:2-3.

16. Garattini S. EMEA: for patients or for industry? Pharmacoeconomics 2005; 23(3):207-208.
17. Brasil. Agência Nacional de Vigilância Sanitária. Gerência de M edicamentos Novos, Pesquisa e Ensaios Clínicos. Posicionamento da Anvisa quanto ao registro de medicamentos novos considerados como me-toos. [site da Internet]. Atualizado em 2004 jun 11. [acessado 2009 nov 17]. Disponível em: http:// www.anvisa.gov.br/medicamentos/registro/ metoos.htm

18. Brasil. Resolução RDC no 28, de 4 de abril de 2007. Dispõe sobre a priorização da análise técnica de petições, no âmbito da Gerência-Geral de M edicamentos da Anvisa, cuja relevância pública se enquadre nos termos desta Resolução. Diário Oficial da União 2007; 5 abr.

19. Organização Pan-Americana da Saúde (Opas). 0 acesso aos medicamentos de alto custo nas Américas: contexto, desafios e perspectivas. Brasília: Organização Pan-Americana da Saúde, Ministério da Saúde, Ministério das Relações Exteriores; 2009. (M edicamentos Essenciais, Acesso e Inovação - THR/EM, 1).

20. Comparative advantages of new drugs: French authorities are not sufficiently demanded. Prescrire International 2005; 14(77):75-79.

21. A look back at 2009: one step forward, two steps back. Prescrire International 2010; 19(106):89-94.

22. HAl Europe/International Society of Drug Bulletins/M edicines in Europe. The European M edicines Agency road map to 2015: independence should be the priority. [site da Internet] [acessado 2010 jun 24]. Disponível em: http://www.prescrire.org/docus/ JointAns_EM AFiveYearPlan2010.pdf

23. Figueiredo TA, Pepe VLE, Osorio de Castro CGS. U m enfoque sanitário sobre a demanda judicial de medicamentos. Physis Revista de Saúde Coletiva 2010; 20(1):101-118.

24. Teixeira M. A bilionária indústria da educação médica continuada nos EUA: mais uma forma de interferência das empresas na relação médico-paciente. Rev Latinoam Psicopat Fund 2009; 12(4):731-742.

Artigo apresentado em 31/05/2010

Aprovado em 21/06/2010

Versão final apresentada em 14/07/2010 
Este artigo sofreu alterações por solicitação do editor em F ev/2011 conforme ERRATA publicada no Volume 16 Número 2 do periódico. (http://www.scielo.br/pdf/csc/v16n2/v16n2a35.pdf) 